\title{
Experimental studies of the $\mathrm{E}_{01}$ leaky wave characteristics in a round dielectric rod
}

\author{
Vasiliy A. Malakhov ${ }^{1,}$, Irina $V$. Malakhova ${ }^{1}$, Artyom $S$. Nechaev $^{1}$, Artyom A. Nikitin $^{2}$, \\ and Yulia $V$. Raevskaya ${ }^{1}$ \\ ${ }^{1}$ Nizhny Novgorod State Technical University n.a. R.E. Alekseev, Nizhny Novgorod, Russia \\ ${ }^{2}$ Branch of FSUE "RFNC-VNIIEF" "NIIIS n. a. Y. E. Sedakov", Nizhny Novgorod, Russia
}

\begin{abstract}
Excitation experiment technique of the $E_{01}$ leaky wave in a round open dielectric waveguide is given. Experimental research results of the $E_{01}$ leaky wave characteristics of a round open dielectric waveguide are presented.
\end{abstract}

Currently, there is a significant interest in devices operating on leaky waves in a world [1-4]. The leaky waves are waves that are excited at frequencies below critical frequencies of surface waves (they are surface waves continuation) of a round open dielectric waveguide. The leaky waves are fast $\left(\mathrm{v}_{\mathrm{p}}>\mathrm{c}\right)$, do not satisfy the radiation condition and have a field exponentially increasing with a radial coordinate increase [5]. One of problems facing researchers is the problem of excitation of the leaky waves, as they quickly fade in propagation direction, resulting in the fields are localized near the source. The paper presents the excitation technique of the $E_{01}$ wave of a dielectric rod and its experimental characteristics.

Figure 1 shows a structural scheme of the experimental setup: 1 - a personal computer; 2 - vector network analyzer OBZOR 804/1; 3 - a researched electrodynamic structure; 4 a measuring element holder with a micrometer; 5 - a loop probe.

For the $E_{01}$ leaky symmetric wave excitation the electrodynamic structure shown in Fig. 1 in the additional window is used. Letters in Fig. 1 are indicated: a - a metal rod with a length of $0.1 \mathrm{~m}$ and a radius of $0.005 \mathrm{~m}, \mathrm{~b}-\mathrm{a}$ round shielded waveguide with a length of $0.15 \mathrm{~m}$ and a radius of $0.03 \mathrm{~m}, \mathrm{c}-\mathrm{a}$ dielectric rod with a length of $0.24 \mathrm{~m}$ and a radius of $0.005 \mathrm{~m}$, the relative permittivity $\varepsilon=2.1$.

At the distance $L_{1}$ along the $\mathrm{z}$ axis the electrodynamic structure is a round coaxial waveguide, at the distance $L_{2}-$ a round shielded two-layer waveguide, and at the distance $L_{3}-$ a round open dielectric waveguide.

The size of electrodynamic structure elements (Fig. 1) selected in such a way that in the existence range $(5-7 \mathrm{GHz})$ of the $E_{01}$ leaky wave of a round open dielectric waveguide the influence of the $H E_{11}$ main wave and the higher type waves on the electric field structure will be minimal, and the $E_{01}$ leaky wave will have minimal attenuation.

In a coaxial waveguide, the $T$-wave is excited, as the field structure of this wave is similar to the field structure of the $E_{01}$ symmetric wave of a round shielded two-layer waveguide, in the area $L_{2}$ (Fig. 1) in addition to the $H E_{11}$ main wave, the $E_{01}$ symmetric

*Corresponding author: physics@nntu.ru 


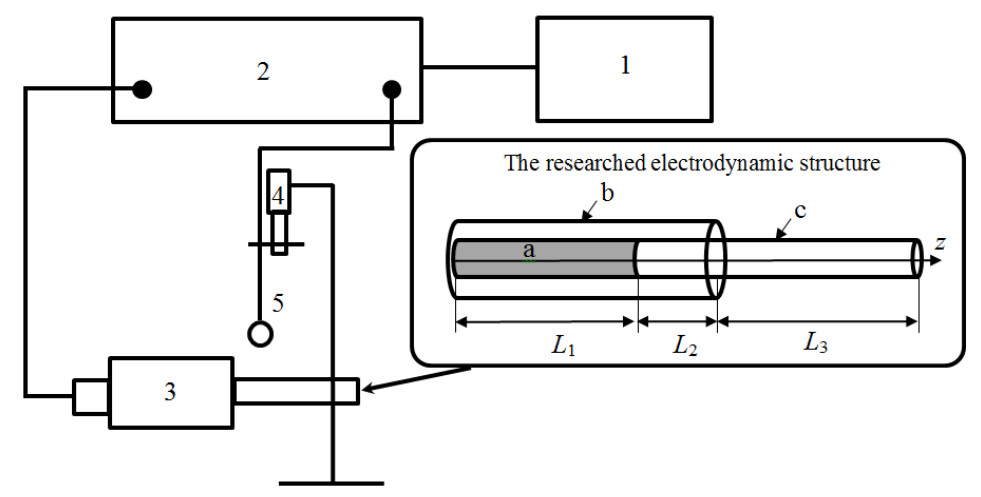

Fig. 1. The structural scheme of the experimental setup.

wave of a round shielded two-layer waveguide and other higher type waves are excited. Modeling and calculations in the computer-aided design system ANSYS HFSS showed the part of energy transferred by the $E_{01}$ symmetric wave is much more than the energy transferred by the other higher type waves. The main part of energy in a round shielded two-layer waveguide is transferred by the $H E_{11}$ wave and the $E_{01}$ symmetric wave, so in a round open dielectric waveguide the main part of energy is transferred by the $H E_{11}$ and $E_{01}$ waves of a round open dielectric waveguide, into which on the $L_{3}$ section the researched electrodynamic structure passes (Fig. 1).

The dielectric $\operatorname{rod}(\varepsilon=2.1)$ forming the open dielectric waveguide at the area $L_{3}$ is a continuation of the rod forming the central layer of the round shielded two-layer waveguide at the area $L_{2}$ (Fig. 1).

The field structure of the $E_{01}$ symmetric wave of a round shielded two-layer waveguide is similar to the field structure of the $E_{01}$ symmetric wave of an open dielectric waveguide, therefore, the $E_{01}$ wave is excited in an open dielectric waveguide in addition to the $H E_{11}$ main wave.

In an open dielectric waveguide, only one $H E_{11}$ surface wave propagates in the existence range of the $E_{01}$ leaky wave, so these two waves have a main influence on a field distribution.

We present the experimental researches results of the effect on a distribution of electric field of the $E_{01}$ leaky wave and the $H E_{11}$ main wave.

The power of $1 \mathrm{~mW}$ was supplied to the input of the researched structure. Power measurements were carried out at a distance of $3 \mathrm{~mm}$ from the two-layer shielded waveguide end using a rigid coaxial cable with the loop probe. The coaxial cable is fixed to the micrometer, by means of which the loop probe is moved along the radial coordinate $r$ (Fig. 2) and field measurements are carried out when the probe moving along the longitudinal coordinate $z$ of the researched structure (Fig. 1) and turning it around the dielectric rod.

Figure 2 shows the dependence of the normalized power flux density on a distance to the dielectric waveguide (on the radial coordinate $r$ ), measured at different frequencies: $1-6.3 \mathrm{GHz}$ (circles), $2-7.5 \mathrm{GHz}$ (stars), $3-8 \mathrm{GHz}$ (triangles).

Figure 2 demonstrates that at a frequency of $6.3 \mathrm{GHz}$, the field structure is determined by the leaky wave, as there is the power flux density increase with a distance increasing to some point. The further power flux density decrease with a distance increase is explained by the fact that the energy flows from the rod having a finite length [6]. At frequencies of $7.5 \mathrm{GHz}$ and $8 \mathrm{GHz}$, the electric field structure is determined by the $H E_{11}$ surface wave of an open dielectric waveguide. The electric field exponentially fades along the radial component. This behavior of the electric field is the characteristic of surface waves. 


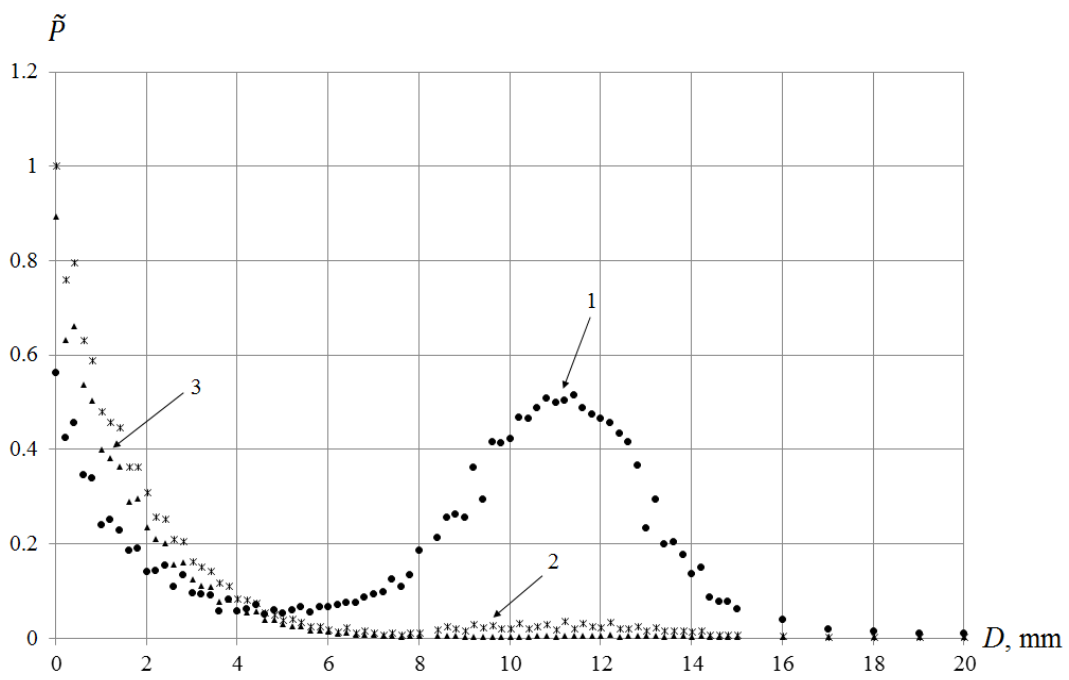

Fig. 2. The dependence of the normalized power flux density on a distance to the dielectric waveguide.

Figure 3 shows the dependence of the normalized power flux density on the longitudinal coordinate $z$ at frequencies: $1-6.3 \mathrm{GHz}$ (circles), $2-8 \mathrm{GHz}$ (triangles).

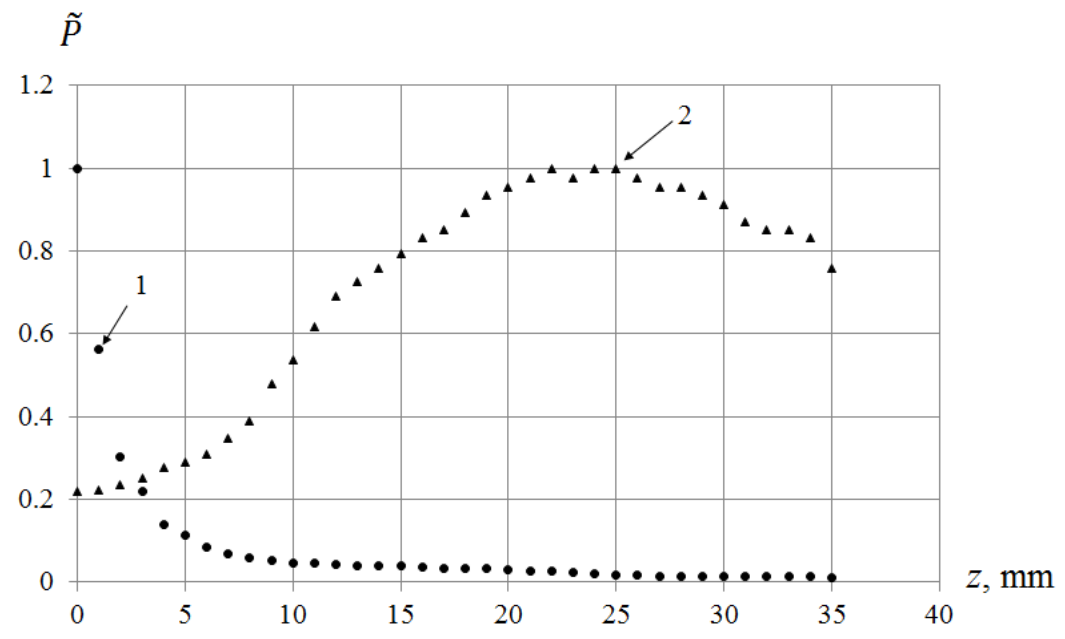

Fig. 3. The dependence of the normalized power flux density on the longitudinal coordinate $z$.

Figure 3 demonstrates that at a frequency of $6.3 \mathrm{GHz}$ there is an exponential attenuation of the electric field, which is typical for the leaky wave, at a frequency of $8 \mathrm{GHz}$ the field has maximums and minimums, which is typical for the surface wave propagating along the dielectric rod and reflecting from the open end in the opposite direction.

The dependence of the power flux density on the radial coordinate at different values of azimuthal angles was also measured (the loop probe rotated around the longitudinal axis $z$ of the dielectric rod (Fig. 1)) at a frequency of $6.3 \mathrm{GHz}$. The measurements showed the measured power at different azimuth angles numerically coincide with each other. Therefore, at a frequency of $6.3 \mathrm{GHz}$ the field structure is symmetrical, that is, the $E_{01}$ symmetric leaky wave of an open dielectric waveguide has the greatest influence on the field structure. 
With a help of a horn antenna at a frequency of $6.3 \mathrm{GHz}$, measurements of the longitudinal and transverse radiation power were made, it showed that in the radial direction the radiation power is $20 \mathrm{~dB}$ more than in the longitudinal direction. This suggests that at a frequency of $6.3 \mathrm{GHz}$ energy radiation occurs in the radial direction, which is also characteristic of leaky waves.

Experimental researches showed the $E_{01}$ symmetric leaky wave is excited in the researched electrodynamic structure at a frequency of $6.3 \mathrm{GHz}$, its characteristics allow making predictions on constructing transverse radiation antennas and antenna irradiators.

\section{References}

1. G. Valerio, S. Paulotto, P. Baccarelli, et al., IEEE Trans. Antennas Propag., v. 63 (6), pp. 2396-2411 (2015)

2. W. Fuscaldo, S. Tofani, D. Zografopoulos, et al., IEEE Trans. Antennas Propag., v. 66 (3), pp. 1169- 1178 (2018)

3. B. Tierney, A. Grbic, IEEE Trans. Antennas Propag., v. 66 (4), pp. 2143-2146. (2018)

4. S. Sengupta, D. Jackson, S. Long, IEEE Trans. Microw. Theory Techn., v. 66 (3), pp. 1181-1191 (2018)

5. A.S. Raevskii, S.B. Raevskii, Complex waves (Radiotekhnika, Moscow, 2010)

6. H.G. Unger, Planar and fiber optical waveguides (Mir, Moscow, 1988) 